\title{
Shedding light on dark chemical matter
}

DCMC hits
are less
promiscuous
but can
interact with
their targets
with high
potency

In each chemical library used for high-throughput screening, there may be compounds that have been screened many times but have never shown biological activity, which may have been assumed to be inert. Now, Wassermann et al. show that these so-called 'dark chemical matter' compounds (DCMCs) can exhibit potent, specific activity, and thus may be a good source of lead molecules.

The authors investigated 234 Novartis assays and 429 PubChem assays (associated with the US National Institutes of Health Molecular Libraries Program) and identified all the screened molecules that had never shown biological activity. 803,990 Novartis molecules and 363,598 PubChem molecules had been screened more than 100 times - of these, 112,872 (14\%)

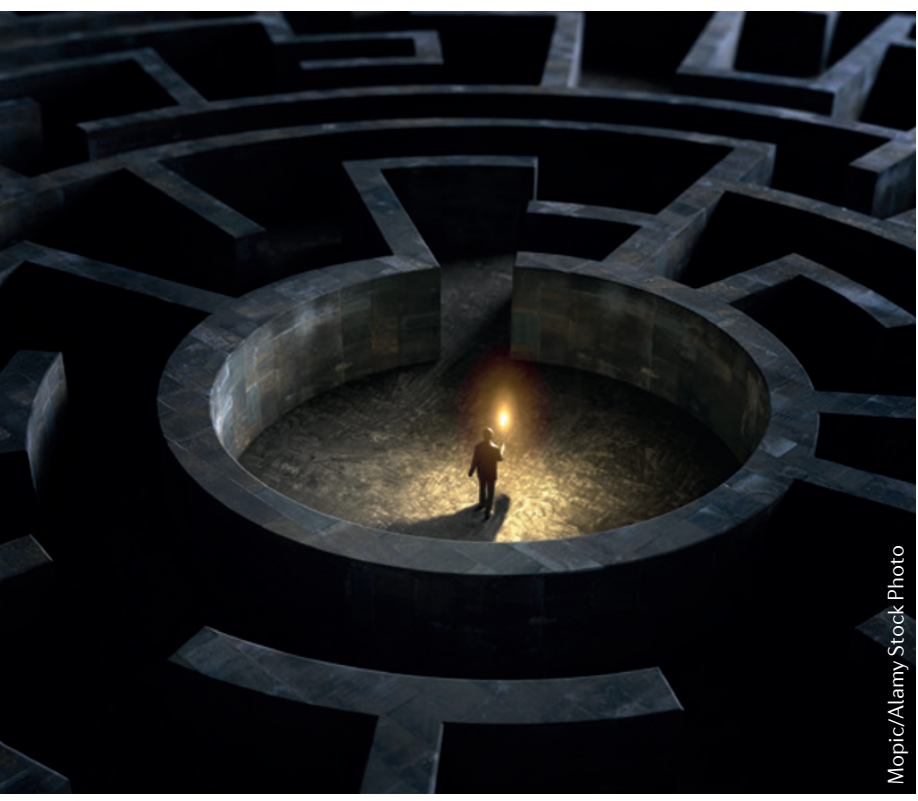

of the Novartis compounds and 131,726 (36.2\%) of the PubChem compounds were consistently inactive and so were defined as DCMCs.

There were approximately tenfold more DCMCs than would be predicted if activities in the assays were distributed randomly across all compounds, suggesting that there might be features of DCMCs that make them less likely to show biological activity. The assay panels featured biochemical as well as cell-based assays, excluding the possibility that the DCMCs were just cell-impermeable, and there were no particular problems of purity or quality among DCMCs. Interestingly, DCMCs were more soluble and less hydrophobic, and tended to have fewer aromatic rings than active compounds (ACTCs).

To further investigate potential differences between DCMCs and ACTCs, the authors mapped these compounds in chemical space using 'extended chemical fingerprints'. Importantly, although some regions in chemical space were heavily populated by DCMCs, they were also interspersed with ACTCs. The observation that DCMCs have active 'neighbours' led the authors to hypothesize that DCMCs are not biologically inert but may appear so in most assays at typical screening concentrations in the low micromolar range because they interact with fewer targets than do other compounds in the screening collection.

Next, the authors examined the performance of DCMCs and ACTCs in 34 further Novartis assays (separate from the first 234). Hit rates of DCMCs in these assays were lower than for ACTCs, even when tested at higher concentrations, but dose-response analysis of a subset of these hits revealed that some of these DCMCs showed submicromolar potency. Thus, DCMC hits are less promiscuous but can interact with their targets with high potency.

Last, the authors screened 374 DCMCs for activity in inhibiting the growth of Saccharomyces cerevisiae. Of these, one compound (compound 1) in particular was found to have potent antifungal activity (with a half-maximal inhibitory concentration of $9.7 \mu \mathrm{M}$ ). Screening of yeast strains haploinsufficent for different proteins indicated that compound 1 may inhibit the protoporphyrinogen oxidase Hem14. Intriguingly, compound 1 had no overt effects on mammalian cells and showed only one interaction with targets commonly associated with adverse drug reactions, suggesting it to be specific for the yeast target. Compounds structurally related to compound 1 exhibited strong antifungal activity against Cryptococcus neoformans, which can cause fungal meningitis and encephalitis.

The study provides evidence that DCMCs are generally not inert, but rather may have more-specific activity than do ACTCs, making them potentially attractive lead molecules.

Natasha Bray

ORIGINAL RESEARCH PAPER Wassermann, A. M. et al. Dark chemical matter as a promising starting point for drug lead discovery. Nat. Chem. Biol. http://dx.doi.org/10.1038/nchembio.1936 (2015) 